\title{
Identificação de fatores de predisposição aos transtornos alimentares: anorexia e bulimia em adolescentes de Belo Horizonte, Minas Gerais
}

\section{I dentification of factors of predisposition for eating disorders: anorexia and bulimia in adolescents of Belo Horizonte, Minas Gerais}

\author{
Nádia Laguárdia de Lima* \\ Universidade Federal de Minas Gerais, Belo Horizonte, Minas Gerais, Brasil
}

\section{Carla de Oliveira Barbosa Rosa**}

Universidade Federal de Viçosa, Viçosa, Minas Gerais, Brasil

\section{J osé Francisco Vilela Rosa***}

Centro Universitário Newton Paiva, Belo Horizonte, Minas Gerais, Brasil

\begin{abstract}
RESUMO
Este trabalho tem como objetivo apresentar o resultado de uma pesquisa interdisciplinar que buscou identificar os fatores de predisposição aos transtornos alimentares, especificamente anorexia e bulimia, em adolescentes do sexo feminino de Belo Horizonte/MG. Foi utilizado como método de pesquisa um estudo transversal, com amostras aleatórias simples. Foram entrevistadas 227 adolescentes do sexo feminino entre 18 e 19 anos, cursando o primeiro semestre de vários cursos de graduação, de diferentes faculdades, privadas e públicas, utilizando-se de formulários padrões, BITE (Teste de Investigação Bulímica de Edimburgo) e o EAT 26 (Teste de Atitudes Alimentares Resumido). O resultado da pesquisa apontou uma predisposição elevada na população estudada à anorexia e bulimia, que pode ser analisada a partir dos efeitos da incidência da cultura da imagem sobre as adolescentes na contemporaneidade.
\end{abstract}

Palavras-chave: Anorexia, bulimia, epidemiologia.

\begin{abstract}
This study aims at presenting the result of an interdisciplinary research that tried to identify the predisposing factors to eating disorders, specifically anorexia and bulimia in female adolescents in Belo Horizonte/MG. A crosssectional research method was used in this study with simple random samples. We interviewed 227 female adolescents between 18 and 19 years old, attending the first semester of several undergraduate courses in different colleges, private and public, using standard forms, BITE (Bulimic Investigatory Test, Edinburgh) and a short version of EAT- 26 ( Eating Attitudes Test ). The survey results showed a high predisposition in this population to anorexia and bulimia, which can be analyzed from the effects of the incidence of culture on the image in contemporary adolescents.

Keywords: Anorexia, bulimia, epidemiology.
\end{abstract}




\section{I ntrodução}

Os transtornos alimentares são patologias de grande importância clínico-social, por apresentarem significativos graus de morbidade e mortalidade, sendo cada vez mais foco da atenção dos profissionais da área de saúde. Dentre os transtornos alimentares, a anorexia e a bulimia tem despertado especial interesse de pesquisadores e clínicos, pelo enorme desafio que essas patologias representam para o campo da saúde.

Com relação à sua etiologia, embora muitos pesquisadores indiquem a existência de fatores genéticos ligados à manifestação dessas enfermidades, é desconhecida a dimensão de sua contribuição para a irrupção da doença. A maioria dos pesquisadores considera a dimensão multifatorial na etiologia dos transtornos alimentares, em especial, dos fatores socioculturais e psíquicos (CORDÁS; OLIVEIRA; FIGUEIREDO; DIDONE, 2007).

Alguns autores consideram que a incidência de transtornos alimentares (TA) praticamente dobrou nestes últimos 20 anos (DUNKER; PHILIPPI, 2003). Especificamente em relação à anorexia, o número de casos novos por ano teve um aumento constante entre 1955 e 1984 em adolescentes de 10 a 19 anos. A prevalência de anorexia nervosa (AN) varia de $2 \%$ a $5 \%$ em mulheres adolescentes e adultas (DUNKER; PHI LIPPI, 2003). Segundo a American Dietetic Association (ADA), os transtornos alimentares são mais frequentes em mulheres adultas e jovens, entre 18 e 30 anos, afetando 3,2\% da população (citado por CORDÁS et al., 2007). No entanto, a irrupção da doença, na maioria das vezes, acontece no despertar da puberdade (COBELO; GONZAGA; NICOLETTI, 2007). Outros autores, entretanto, apesar de considerarem um aumento no número de casos, não acreditam em uma "epidemia" desses transtornos na atualidade. Para Fernandes (2006) o aumento no número de registros de casos de pessoas com transtornos alimentares ocorreu no momento em que a medicina começou a reconhecer esse tipo de patologia, o que pode ter influenciado os resultados. A autora acredita que o aumento do número de pessoas com transtornos alimentares tenha ocorrido a partir do crescente interesse dos profissionais de saúde e da própria população leiga depois que passaram a receber informações sobre esses distúrbios através dos meios de comunicação (FERNANDES, 2006).

Mesmo que não se possa falar em epidemia de transtornos alimentares na contemporaneidade (FERNANDES, 2006), não se pode desconhecer sua significativa presença na sociedade atual, principalmente entre as adolescentes e jovens do sexo feminino.

Não existe um consenso com relação aos métodos mais adequados para a avaliação desses transtornos. Os instrumentos para a avaliação dos transtornos alimentares (TA) surgiram com a 
necessidade de sistematizar os estudos a partir do estabelecimento e do aprimoramento de seus critérios diagnósticos. De um modo geral, os instrumentos de avaliação são agrupados em, pelo menos, três categorias: 1) questionários autoaplicáveis; 2) entrevistas clínicas e 3) automonitoração (FREITAS; GOVENSTEIN; APPOLINASE, 2002). Esses instrumentos são utilizados tanto para o diagnóstico quanto para a identificação de sinais de predisposição aos transtornos alimentares.

Algumas pesquisas realizadas no Brasil com adolescentes e jovens (KIRSTEN; FRATTON; PORTA, 2009; CONTI; FRUTUOSO; GAMBARDELLA, 2005; VILELA; LAMOUNIER; FILHO; BARROS NETO; HORTA, 2004) têm revelado significativa predisposição na população estudada aos transtornos alimentares. Segundo Kirsten; Fratton e Porta (2009), a prevalência de bulimia nervosa clinicamente significativa em estudantes universitárias é muito maior do que a observada na sociedade, correspondendo a, aproximadamente, 4,0\%.

\section{Revisão de Literatura}

\subsection{Conceituando a adolescência}

A maior incidência de transtornos alimentares na adolescência permite considerar esta fase da vida como propícia ao desencadeamento da doença. A adolescência é um conceito social, uma criação das sociedades modernas, como resultado do prolongamento da escolarização. O termo é utilizado na nossa cultura para definir um estágio da vida entre a infância e a fase adulta, como um tempo de "preparação" para a entrada na fase adulta. Alguns autores (CIRINO, 2001) acreditam que há um prolongamento da adolescência na atualidade devido a uma certa "idealização" dessa fase da vida. Esta idealização existe porque a cultura ocidental tem como um dos valores fundamentais a juventude, equivalendo-a à felicidade. A felicidade plena seria a possibilidade de "gozar" sem restrições. O adolescente é visto como aquele que tem o "corpo ideal" (a beleza da juventude) e o acesso à vida sexual, sem ter que assumir as responsabilidades da vida adulta. Nessa perspectiva, a adolescência seria a fase ideal da vida. Se a adolescência não pode ser bem delimitada cronologicamente em função das variações culturais, o termo "puberdade" apresenta uma conotação mais precisa, por sua característica biológica. Ele é marcado por transformações fisiológicas e hormonais que levam à conquista do corpo adulto.

De acordo com a teoria psicanalítica, o termo adolescência pode ser utilizado para conceituar as consequências psíquicas da puberdade, ou seja, um tempo lógico para que o sujeito possa construir uma 
significação própria para essas mudanças fisiológicas e hormonais. São conhecidas as mudanças marcantes na fisiologia e bioquímica no tempo da puberdade, com um acúmulo pronunciado de gordura, especialmente nas meninas. Uma série de mudanças sexuais e fisiológicas ocorre em função de alterações no nível dos hormônios. A hipófise produz dois hormônios: o LH (Lutotrófico) e o FSH (hormônio folículo estimulante), provocando a rápida transformação corporal e o surgimento dos caracteres sexuais (GREEN, 1997). O ganho de gordura durante a adolescência cessa e até se reverte nos homens, enquanto nas mulheres continua a aumentar, chegando a um índice de gordura corporal de $27 \%$ aos 16 anos (DUNKER; PHILIPPI, 2003). As marcantes mudanças fisiológicas e hormonais que ocorrem nesta fase da vida produzem efeitos psíquicos sobre os sujeitos (FREUD, 1974/1905). Na adolescência o sujeito se vê confrontado com a necessidade de assumir uma posição sexuada, de buscar novas referências de identificação que não sejam os pais, de fazer uma escolha profissional e ingressar no mundo social. Essas exigências despertam angústia nos jovens, que se sentem despreparados para os novos papéis que devem assumir na vida.

A preocupação com o corpo ocupa um lugar central na adolescência, quando os jovens precisam reconstruir a imagem corporal e conquistar uma identidade sexual. Esse período da vida é, pois, marcado por crises, que propiciam o surgimento de sintomas alimentares. Podemos dizer que os sintomas alimentares revelam dificuldades na transição do corpo infantil para o corpo adulto.

A cultura atual corrobora para a existência de uma maior preocupação com relação à imagem corporal (DEBORD, 1992), afetando especialmente as meninas adolescentes. $O$ ganho de gordura no tempo da puberdade especialmente nas meninas pode gerar grande preocupação nos familiares e na própria adolescente. A idealização da adolescência na nossa cultura favorece um interesse precoce das crianças em adquirir o "corpo magro", cultuado pela mídia, levando à prática de dietas e atividades físicas, muitas vezes sem orientação profissional. Assim, fatores culturais se associam aos fatores subjetivos levando ao desencadeamento dos transtornos alimentares na adolescência, em especial, a anorexia e a bulimia.

Diversos profissionais da saúde marcam a importância de se intervir - mais cedo possível para que o tratamento dos pacientes com distúrbios alimentares seja eficaz (BUCARETCHI, 2003; CORDÁS et al., 2007). No entanto, a dificuldade em reconhecer os sinais clínicos da doença e a recusa do próprio paciente em aceitar um tratamento, levam ao agravamento da doença, com todos os riscos que ela envolve.

A história clínica destes pacientes revela a presença de alguns sinais antes do estabelecimento do diagnóstico médico de anorexia e bulimia. Segundo Cordás et al. (2007), os transtornos alimentares 
apresentam fatores predisponentes, precipitantes e mantenedores. Como fatores predisponentes, os autores descrevem: genéticos (doenças psiquiátricas na família), biológicos (tendência à obesidade e outros), neurológicos (alterações nos sistemas de neurotransmissão), personalidade (baixa autoestima, perfeccionismo, rigidez ou impulsividade), familiares (dinâmicas familiares marcadas por superproteção, rigidez excessiva, falta de afetos), socioculturais (supervalorização da magreza e grande oferta de alimentos) e outros. Como fatores precipitantes: dieta para perder peso, perdas ou separações. Como fatores mantenedores: alterações neuroendócrinas, distorção da imagem corporal, distorções cognitivas, práticas purgativas, alterações psicológicas e fatores socioculturais. A identificação desses fatores pode ajudar familiares, educadores e outros profissionais que trabalham com adolescentes a buscar uma intervenção clínica a tempo.

\subsection{Conceituando a anorexia e a bulimia}

\subsubsection{Uma leitura biológica}

A anorexia e a bulimia são transtornos alimentares caracterizados por um padrão de comportamento alimentar gravemente perturbado, um controle patológico do peso corporal e por distúrbios da percepção do formato corporal. Está presente, na anorexia nervosa, um inexplicável medo de ganhar peso ou de tornar-se obeso, mesmo estando abaixo do peso, ou mais intensamente, uma supervalorização da forma corporal como um todo ou de suas partes, classicamente descrito como distorção da imagem corporal (CORDÁS, 2004).

Os critérios diagnósticos dos transtornos alimentares têm sido amplamente estudados nos últimos 30 anos. São descritos como transtornos e não como doenças por ainda não se conhecer bem sua etiopatogenia. (CLAUDINO; BORGES, 2002). Os atuais sistemas classificatórios de transtornos mentais, DSM-IV (Diagnostic and Statistical Manual, IV edition) e CID 10 (Classificação Internacional de Doenças, 10 을 edição), ressaltam duas entidades nosológicas principais dos transtornos alimentares: Anorexia Nervosa (NA) e a Bulimia Nervosa (BN). Os dois transtornos apresentam algumas características comuns, como a preocupação excessiva com o peso e com a forma corporal.

Como sintomas mais comuns, as anoréxicas apresentam manutenção do peso corporal inferior a $85 \%$ do que é considerado adequado para a estatura e a idade, medo mórbido de engordar, alteração da percepção da imagem corporal, distúrbios menstruais, desmineralização óssea, perda de massa muscular e gordura 
corporal, irregularidades digestivas, arritmias cardíacas, desidratação, intolerância ao frio, cabelos finos e fracos, entre outros (ANDRADE; BOSI, 2003).

As pacientes anoréxicas, por sua inadequada ingestão de alimentos, em geral apresentam quadro de desnutrição proteico-calórica em diferentes graus. $\mathrm{Na}$ avaliação do IMC as pacientes apresentam medianas abaixo do valor mínimo da faixa de normalidade, sendo este achado associado a quadros carenciais graves de vitaminas e minerais, entre os quais o cálcio. Portanto, uma das consequências nutricionais mais importantes na evolução da AN é a carência de cálcio. Em condições de baixa ingestão prolongada de cálcio, este passa a ser retirado de estruturas que o contêm, como os ossos, podendo provocar prejuízos de graus variáveis na sua densidade mineral (SANTOS; RIBEIRO; SANTOS; ROSA E SILVA; SÁ, 2004).

$A$ restrição energética afeta $O$ consumo de macronutrientes. A aversão aos carboidratos e gorduras é evidente, enquanto o consumo de proteínas normalmente está dentro da porcentagem que deve ser ingerida, apesar de o total ser inadequado e sua utilização comprometida pela ingestão energética insuficiente (DUNKER; PHI LIPPI, 2003).

A anorexia é uma patologia "silenciosa", que se instala de maneira lenta e progressiva, dificultando o estabelecimento de um momento preciso de seu início. Lasègue (1884) descreve a evolução desse transtorno em três fases principais. Na primeira, acontece o desencadeamento e a instalação do transtorno, quando o sujeito pode deixar de se alimentar como resposta a um evento importante como um luto, um acidente, uma separação, uma decepção, uma dor de cabeça. A repugnância em se alimentar não é superada, mas continua e evolui de forma lenta e progressiva.

$\mathrm{Na}$ segunda fase, a depressão, o desconforto e o mal-estar da primeira fase dão lugar a um "certo equilíbrio", um relativo otimismo e uma sensação de "bem-estar". A dieta alimentar autoimposta se torna cada vez mais rígida, chegando a alcançar graves restrições alimentares. Os sintomas clínicos de anemia ou de qualquer outra doença orgânica ainda não se mostram presentes e os pacientes costumam ficar bem dispostos e alegres. O único sintoma aparente é a perda de peso. No entanto, nesse momento os familiares começam a se preocupar, se mostram incomodados e inquietos com a despreocupação do anoréxico diante do próprio emagrecimento. Alguns passam, então, a adverti-los sobre esses sintomas e podem até procurar ajuda profissional. Contudo, essa reação da família pode provocar um aumento de resistência por parte do anoréxico.

O terceiro período da evolução do distúrbio é marcado por uma incapacidade orgânica. As restrições alimentares afetam o corpo, que começa a apresentar suas falhas, tais como a amenorréia, a retração das paredes abdominais, a diminuição da elasticidade acompanhada 
da opacidade da pele e a constipação. Esta ordem de evolução pode apresentar divergências, havendo a necessidade de uma avaliação individualizada.

A anorexia nervosa (AN) apresenta significativa morbidade, estando a amenorréia prolongada presente na maioria dos casos. Por acometer principalmente adolescentes, incide em período crítico de formação do esqueleto e, portanto, uma das complicações mais graves é a redução da massa óssea. A incidência de osteopenia pode chegar a $90 \%$ entre estas jovens, sendo que um terço delas tem osteoporose (SANTOS et al., 2004).

A principal característica da bulimia é a ingestão alimentar incontrolável, como um "acesso" ou uma "crise bulímica", seguida por algum tipo de purgação. Apresenta-se uma prática de consumo de alimento muito além do necessário e acompanhada de sensações de perda de controle, levando a bulímica a condutas extremas como provocação de vômitos, uso excessivo de laxantes, e/ou diuréticos.

As crises bulímicas podem acontecer por horas a fio ou várias vezes no mesmo dia. O aumento no número de crises pode provocar sérias consequências no organismo, tais como: dor na garganta e no estômago, irregularidades menstruais, episódios de sudorese, debilidade e sonolência e, em alguns casos, pode produzir também alterações dentárias (perda do esmalte do dente por efeito químico do ácido gástrico), hipotensão arterial e arritmias cardíacas (FERNANDES, 2006).

A grande dificuldade no diagnóstico da doença ocorre pela sua falta de "visibilidade", já que, diferentemente da anorexia, não há uma perda significativa de peso na bulimia (FERNANDES, 2006).

\subsubsection{Uma leitura social}

A história da anorexia e bulimia nervosa revela que esses transtornos não são contemporâneos. De acordo com Weinberg (2005), na época em que viveu Santa Catarina de Siena, existiu uma verdadeira epidemia da anorexia santa. As Santas do século XIII chegaram ao extremo de recusar totalmente qualquer alimento. Eram chamadas de "santas anoréxicas". O quadro era acompanhado de perfeccionismo, autoinsuficiência, rigidez no comportamento, insatisfação consigo própria e distorções cognitivas.

Na bulimia, o termo já era usado séculos antes de Cristo. Hipócrates o empregava para designar uma fome doentia, diferente da fome fisiológica (CORDÁS, 2004). Em 1743, James descreve a true boulimus para os episódios de grande ingestão de alimentos e preocupação intensa com os mesmos, seguidos de desmaios.

Os fatores culturais exercem importante influência no surgimento dos transtornos alimentares. Se os transtornos alimentares não são contemporâneos, eles ganham maior visibilidade na atualidade. Essa 
maior visibilidade pode indicar o resultado do aumento de sua incidência, de maior informação sobre essas enfermidades e de sua divulgação na mídia. A cultura atual confere um lugar de destaque à imagem corporal, exaltando a magreza, equivalendo-a ao sucesso e à felicidade. Constata-se que as mulheres, de uma maneira geral, são mais vulneráveis às pressões sociais, econômicas e culturais, associadas aos padrões estéticos.

Pesquisas atestam o conflito vivido pelas mulheres entre o ideal de beleza prescrito pela sociedade atual e o somatotipo da maioria da população. O medo da obesidade faz com que um número cada vez maior de mulheres e cada vez mais novas faça dietas e busque um controle excessivo do peso corporal, através de exercícios físicos em excesso, drogas e laxantes.

Observa-se hoje que o corpo feminino é utilizado de forma incisiva pela publicidade, que através dos diferentes meios de comunicação, invade os lares e alcança as crianças e adolescentes, que passam a aliar a satisfação e a realização pessoais à beleza e à magreza.

Os estudos na área médico-psicológica, de acordo com Ramalho (2005), têm associado o aparecimento dos transtornos alimentares na atualidade a uma exagerada preocupação com o corpo, corroborada pelo ideal social do "corpo perfeito", que preconiza a magreza como padrão estético.

As crenças e valores culturais determinam normas sociais na relação do sujeito com o seu corpo. As práticas de embelezamento, manipulação e mutilação corporais são carregadas de significados simbólicos e ilustram o lugar que o corpo ocupa na cultura. Na cultura capitalista, o corpo adquire o valor de objeto de consumo, que pode ser "moldado, transformado e produzido" segundo os ideais sociais, graças à indústria estética e aos avanços tecnológicos.

Os adolescentes hoje vivem bombardeados por uma exposição maciça de corpos na mídia. Os meios de comunicação em geral promovem imagens perfeitas e inalcançáveis de beleza. As jovens adolescentes inseguras da própria imagem corporal acabam sendo alvos mais fáceis da indústria da estética. No entanto, os fatores culturais não são suficientes para explicar o surgimento da anorexia e bulimia na adolescência.

\subsubsection{Uma leitura psicanalítica}

Entre os estudos psicanalíticos, existe um relativo consenso em atribuir esses sintomas a dificuldades no processo inicial de constituição subjetiva, no momento de formação da imagem corporal. A constituição da imagem corporal se dá nos primeiros meses de vida da criança, através de um processo de identificação com a imagem oferecida pela mãe. O olhar de desejo da mãe confere à criança o reconhecimento da própria imagem unificada no espelho (LACAN, 
1998/1949). Esse processo é fundamental para a construção do narcisismo, que oferece ao sujeito uma identidade imaginária (FREUD, 1974/1914).

Os pacientes com transtornos alimentares apresentam acentuada insatisfação com relação à imagem corporal. Segundo Ramalho (2005), a frágil imagem de si, característica das pessoas que apresentam anorexia e bulimia, muitas vezes decorre do fato de se sentirem atendidas somente em suas necessidades biológicas e não encontrarem acolhidas as suas demandas psíquicas. Muitas vezes não Ihes foi oferecido um olhar de desejo, através do qual pudessem obter um reconhecimento enquanto sujeito.

De acordo com Lacan (1995/1956-57), a anorexia é um comer nada, que é a maneira encontrada pelo sujeito para dizer um "não" à papinha sufocante que a mãe oferece incessantemente. A mãe responde às necessidades da criança com o alimento, confundindo seu cuidado com o dom de amor. Diante da angústia de não saber o que o bebê está lhe demandando, por não entender o que o choro do bebê significa, ela responde com o alimento. O objeto de amor é reduzido à necessidade. A mãe sufoca a criança com o alimento, só restando à criança recusá-lo, para salvar o seu desejo.

Diversos autores enfatizam dificuldades nas relações primordiais na etiologia desses transtornos alimentares. Segundo Bidau (1998), a adolescente anoréxica apresenta grandes dificuldades com relação à figura materna, encontrando na recusa oral a única forma de se opor e de "se fazer olhar" pela mãe. Assim, o sintoma alimentar revela sua ambiguidade: a adolescente tenta se separar da mãe e, paradoxalmente, se manter ligada a ela. Para o autor, o corpo gordo é objeto de angústia da anoréxica: engordar é perder o domínio do eu, é rebaixar-se à posição de objeto, mantendo-se numa posição de alienação à mãe.

Estes transtornos alimentares aparecem justamente no momento em que surgem as transformações corporais, momento da puberdade, quando a irrupção do sexual surge como um enigma para o sujeito. $\mathrm{Na}$ adolescência, as rápidas modificações corporais e a "explosão hormonal" levam o jovem a buscar construir uma significação para esse real incontrolável. O corpo se transforma a cada dia, exigindo do sujeito a reconstrução da imagem corporal. Há um abalo das identificações. Os pais, até então referências de identificação, não servem mais como modelos. Os jovens buscam novas referências de identificação. Há o questionamento do próprio desejo e da sexualidade. Freud (1974/1905) ressalta, no tempo da puberdade, dois importantes trabalhos psíquicos, o de separação dos pais e o de escolha de uma posição sexuada. Diversos autores têm destacado, nos adolescentes anoréxicos, as dificuldades com relação a esses dois trabalhos psíquicos. Constata-se com frequência a recusa à feminilidade e ao corpo sexuado (RAMALHO, 2005). Os transtornos 
alimentares revelam dificuldades da jovem adolescente com a conquista da feminilidade e com a assunção do próprio desejo.

$\mathrm{Na}$ cultura contemporânea, os ideais estéticos de esbelteza reforçam a insatisfação com a imagem corporal, promovendo o aparecimento de sintomas alimentares na adolescência. Para Bleichmar (2000), acompanhamos na atualidade um culto tão intenso às formas que a mulher fica demasiado exposta. A superexposição do corpo leva a mulher a sentir seu corpo invadido pelo olhar do outro. A aparência, para além do nível superficial, toca o íntimo das mulheres. Assim, se as mulheres, por um lado, exibem o corpo-espetáculo, permitindo essa intrusão escópica para não serem excluídas da cultura, por outro lado, temem por sua integridade corporal. Há uma perda da dimensão da fantasia, já que tudo sobre a sexualidade está exposto e dito.

A recusa ou os excessos na alimentação desvelam conflitos de ordem psíquica, que devem ser escutados como um apelo, um pedido de socorro. Uma vez instaurada a anorexia ou a bulimia, há uma série de prejuízos à saúde, podendo levar o paciente à morte.

\section{Metodologia}

\subsection{Amostra}

A pesquisa foi realizada com 227 adolescentes do sexo feminino, entre 18 e 19 anos de idade, regularmente matriculadas no primeiro semestre de graduação de diferentes cursos em faculdades públicas e privadas de Belo Horizonte. A escolha da idade das alunas obedeceu a dois critérios: a sua localização na fase da adolescência, pois, segundo a Organização Mundial de Saúde (OMS) a adolescência compreende o período entre 10 e 19 anos de idade (CONTI et al., 2005) e o fato de estarem no final da fase da adolescência, pois, a partir dos 18 anos de idade não é mais necessário o consentimento dos pais para a participação na pesquisa, apenas do adolescente.

\subsection{Procedimentos}

As adolescentes foram convidadas, em sala de aula, através da explanação dos objetivos e importância da pesquisa, a participarem do estudo e receberam os esclarecimentos solicitados, por duas alunas bolsistas, sendo uma do curso de Nutrição e outra do Curso de Psicologia. Ao aceitarem participar da pesquisa, as estudantes assinaram um termo de consentimento livre e esclarecido baseado na Resolução 196 de 10/10/1996 do Conselho Nacional de Saúde. Foi entregue um questionário de autopreenchimento para cada estudante, que não continha identificação pessoal. Todas as alunas 
que aceitaram participar da pesquisa responderam a todas as perguntas. Os questionários foram aplicados nas escolas onde os adolescentes estudavam.

Buscamos identificar a prevalência de fatores de predisposição aos transtornos alimentares, anorexia e bulimia, em adolescentes. O estudo antropométrico baseou-se nos dados referidos pelas entrevistadas, não tendo sido possível efetuar a avaliação antropométrica direta. Esta pesquisa científica foi conduzida dentro de padrões éticos exigidos pela Comissão Nacional de Ética em Pesquisa / Conselho Nacional de Saúde / Ministério da Saúde (CONEP/CNS/MS) e financiada pela FUNADESP (Fundação Nacional de Desenvolvimento do Ensino Superior Particular).

\subsection{I nstrumento}

Foi escolhido como método de pesquisa um estudo transversal, com amostras aleatórias simples. Foram utilizados o Teste de Investigação Bulímica (BITE) e o Teste de Atitudes Alimentares Resumido (EAT 26). Ambos avaliam a presença de comportamentos de risco alimentar, principalmente Anorexia Nervosa e Bulimia. O Bite avalia especificamente comportamentos bulímicos como a ingestão excessiva de alimentos e utilização de métodos purgativos para compensar os episódios exagerados de alimentação. Constitui-se de 33 questões dicotômicas, pontuadas entre zero e um $(0-1)$, podendo totalizar 30 pontos, sendo que 10 é o ponto de corte que indica a presença de comportamento alimentar de risco. Respostas positivas ("sim") indicam a presença do sintoma, sendo pontuadas em 1 e respostas negativas ("não") indicam a ausência, valendo 0 pontos. Cinco questões possuem pontuação inversa, o "não" vale 1 e o "sim" vale 0. Este instrumento foi traduzido e adaptado para o Brasil por Cordáz (1993), apresentando boas qualidades psicométricas de validade e confiabilidade.

Já o EAT-26 é um instrumento desenvolvido por Garner, Olmested e Polivy (1983) para avaliar sintomas de Anorexia Nervosa. Foi traduzido e validado para o Brasil por Bighetti (2003), tendo apresentado bons indicadores de consistência interna (Alpha de Cronbach) e validade. Trata-se de uma escala com 26 itens com opções de resposta do tipo Likert de 6 pontos. Confere-se pontos variando de 0 a 3 assim divididos: "sempre"=3 pontos, "muitas vezes" $=2$ pontos, "às vezes" $=1$ ponto, "poucas vezes, quase nunca e nunca" = 0 pontos. A pontuação pode variar de 0 a 78 pontos, sendo que o ponto de corte utilizado como indicador positivo da existência de distúrbio alimentar é igual a 21. Quanto maior a pontuação, maior o risco da presença de transtorno alimentar. 


\section{Resultados e discussão}

Das 227 adolescentes entrevistadas, o EAT + foi de $20,7 \%$ e o BEAT + foi de $15,4 \%$, significando uma predisposição elevada na população estudada à anorexia e bulimia. Os dados obtidos estão de acordo com os resultados apresentados em outras pesquisas similares (KIRSTEN et al., 2009; CONTI et al., 2005; VILELA et al., 2004). Estudos nacionais feitos com estudantes de Nutrição mostram altas prevalências de comportamentos sugestivos de TA quando aplicado o EAT-26. Nas pesquisas realizadas por Fiates e Salles (2001) em Florianópolis, e Stipp e Oliveira (2003) em Piracicaba, encontraramse, respectivamente, prevalências de $22,2 \%$ e $18 \%$. Conforme os autores relatam, prevalências de EAT+ maiores de $20 \%$ são bastante preocupantes (GARCIA; CASTRO; SOARES, 2010).

Os dados levantados em nossa pesquisa são bastante relevantes. Foram detectados os seguintes fatores precipitantes e mantenedores da doença (CORDÁS et al., 2007): dieta para perder peso, distorção da imagem corporal, práticas purgativas, alterações psicológicas e fatores socioculturais. As pessoas com o diagnóstico de anorexia e/ou bulimia apresentam um histórico de grande preocupação com relação ao peso corporal, problemas alimentares desde a infância e experiências frequentes com dietas e jejuns sem orientação de um profissional especializado.

\subsection{Insatisfação com a imagem corporal}

Das jovens entrevistadas, $23 \%$ mostram-se sempre preocupadas com o desejo de serem mais magras, $30 \%$ estão sempre apavoradas com o excesso de peso, $13 \%$ declaram que as outras pessoas as consideram magras demais, o que mostra uma distorção da imagem corporal nestas adolescentes, já que a percepção do próprio corpo não coincide com a percepção que as outras pessoas tem de seus corpos, $28 \%$ preocupam-se sempre com a possibilidade de ter gordura em seu corpo, $85 \%$ dizem que o pensamento sobre a possibilidade de tornarem-se gordas as apavora.

\subsection{Distúrbios alimentares}

Dentre os fatores precipitantes na etiologia dos transtornos alimentares, a dieta para emagrecer é o fator mais frequente (CONTI et al., 2005). De acordo com os dados coletados em nossa pesquisa: $27 \%$ das estudantes declaram já ter tido algum tipo de problema alimentar, $47 \%$ relatam já ter jejuado por um dia inteiro, $26 \%$ declaram que o seu padrão de alimentação prejudica severamente a sua vida, $26 \%$ afirmam comer sem parar até serem obrigadas a parar 
por sentirem-se mal fisicamente, 74\% afirmam ter tido episódios exagerados de alimentação, $45 \%$ consideram que os seus hábitos alimentares não são normais.

\subsection{Alterações psicológicas}

As adolescentes pesquisadas percebem o descontrole na relação com a comida e um desconforto (angústia) na origem dessa relação: $46 \%$ buscam através da comida aliviar algum tipo de desconforto, 22\% consideram que comem compulsivamente, e $57 \%$ declaram ter preocupação com o fato de não terem controle sobre o quanto comem. As adolescentes pesquisadas apresentam-se constantemente insatisfeitas com relação ao próprio corpo, mesmo quando estão com o peso adequado. Esta insatisfação com a imagem corporal aponta para uma insatisfação "consigo mesma" que, com frequência, desvela conflitos estabelecidos desde a mais tenra infância, no momento inicial de construção da imagem de si, fundada na relação com a figura materna. Uma "escuta clínica" proporciona elementos para a confirmação dessa hipótese, que não pode ser investigada nessa pesquisa. A insatisfação com a própria imagem tem levado algumas das adolescentes pesquisadas à adoção de medidas extremas para alcançar o baixo peso: $7 \%$ das adolescentes pesquisadas utiliza laxantes para auxiliar na perda de peso, 5\% faz uso de vômitos e 4\% de comprimidos.

A Figura 1 ilustra os resultados, em termos percentuais, das respostas das adolescentes entrevistadas de algumas perguntas dos questionários EAT 26 e BITE. 
Nádia Laguárdia de Lima, Carla de Oliveira Barbosa Rosa, J osé Francisco Vilela Rosa Identificação de fatores de predisposição aos transtornos alimentares

Figura 1 - Resultados de algumas perguntas realizadas durante a aplicação dos questionários EAT 26 e BITE, em adolescentes universitários de 18 e 19 anos. do sexo feminino, em Belo Horizonte, MG

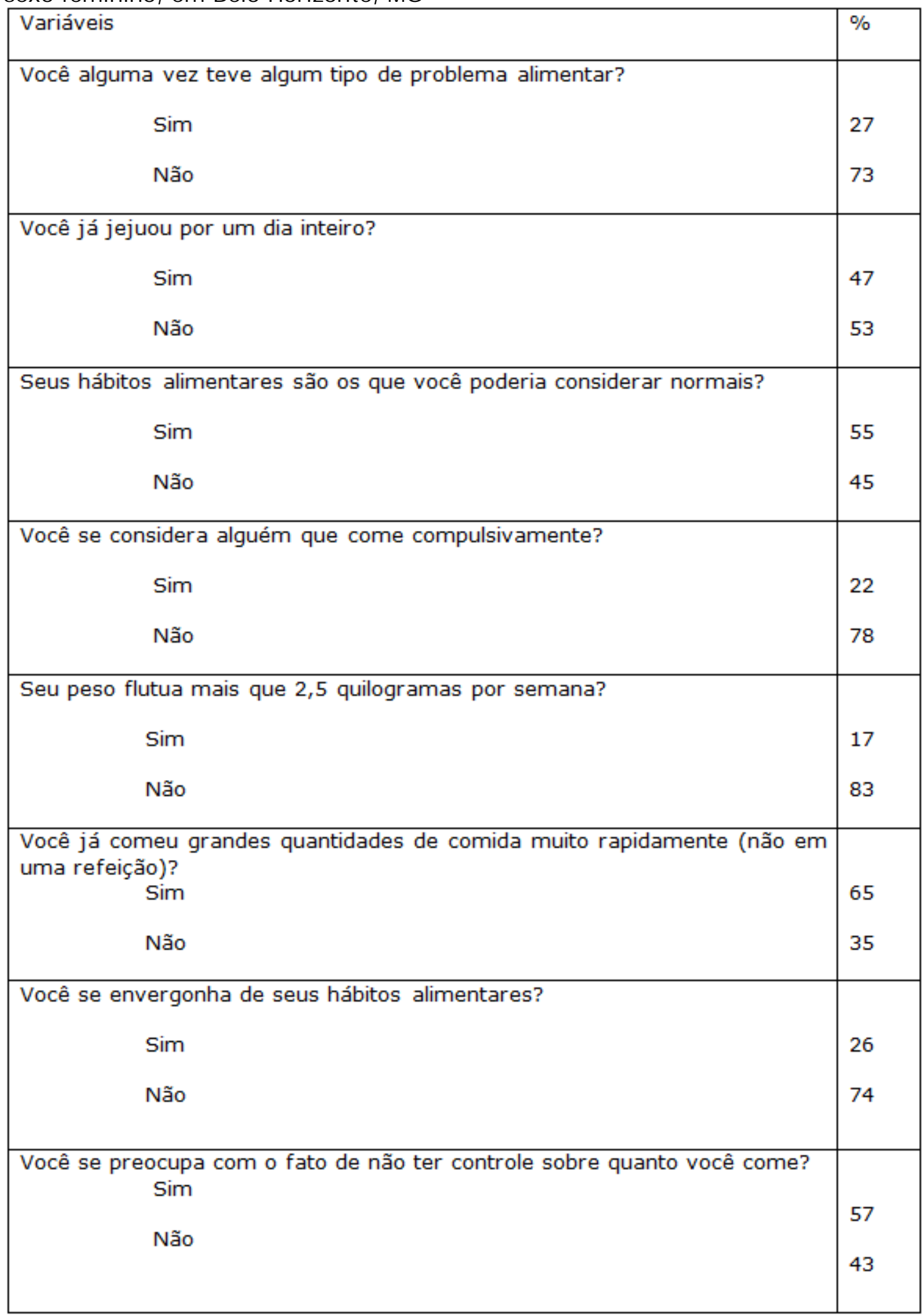

Estud. pesqui. psicol., Rio de Janeiro, v. 12, n. 2, p. 360-378, 2012. 


\section{Considerações finais}

Os resultados obtidos nesta pesquisa são importantes indicadores de fatores predisponentes da anorexia e bulimia na população estudada. Eles sugerem o peso da cultura da esbelteza sobre as jovens adolescentes, que, no momento em que precisam refazer a imagem corporal, se deparam com os ideais estéticos culturais inalcançáveis, constantemente bombardeados pela mídia. No entanto, os fatores socioculturais são insuficientes para explicar o desencadeamento dessas patologias.

As jovens que apresentam anorexia ou bulimia apresentam grande insatisfação com relação à própria imagem corporal. Considera-se que a adolescência é uma fase crítica da vida, quando os jovens se deparam com as exigências de se posicionarem com relação à sexualidade, reconstruirem a imagem corporal, se separarem dos pais e assumirem um lugar na vida social. Os adolescentes precisam utilizar os recursos psíquicos de que dispõem para lidar com as grandes exigências que lhe são impostas nesse momento da vida. Estes recursos foram construídos desde a mais tenra infância e podem se mostrar insuficientes para lidar com as exigências subjetivas de se posicionar diante do sexo e da vida social no tempo da adolescência.

Dada a maior incidência de transtornos alimentares nessa fase da vida, pesquisas sobre o tema tornam-se fundamentais para a elaboração de políticas públicas de saúde voltadas para a prevenção e intervenção junto à população adolescente. Projetos de prevenção nas escolas deveriam ser construídos, visando oferecer espaços de interlocução entre adolescentes e profissionais da saúde. Nesses espaços, seriam abertas possibilidades para discussões e reflexões críticas sobre os valores contemporâneos, em especial, o ideal de magreza na cultura atual e seus efeitos sobre os jovens. Os indicativos para o desenvolvimento de transtornos alimentares não podem ser tomados como um diagnóstico dessas patologias. O diagnóstico só pode ser estabelecido segundo uma avaliação individual, feito por uma equipe interdisciplinar, que deve incluir: avaliação médica, nutricional e psicológica (e/ou psiquiátrica). O reconhecimento dos sinais de risco para o desenvolvimento de transtornos alimentares pode ajudar os profissionais a identificar adolescentes com chance de desenvolver esses transtornos ou que se encontram no estágio inicial da doença, encaminhando-os para os serviços de atendimento para o estabelecimento de um diagnóstico preciso e um tratamento adequado, evitando o agravamento da doença. 


\section{Referências}

ANDRADE, A.; BOSI, M. L. M. Mídia e subjetividade: impacto no comportamento alimentar feminino. Revista de Nutrição, Campinas, v. 16, n. 1, 2003. Disponível em: $<$ http://www.scielo.br/scielo.php?script=sci_arttext\&pid=S1415-

52732003000100012\&lng=pt\&nrm=iso >. Ačesso em: 06 ago. 2007.

BIDAU, E. Anorexia mental, ascese, mística. Uma abordagem psicanalítica. Rio de Janeiro: Companhia de Freud, 1988.

BIGHETT, F. Tradução e validação do Eating Attitudes Test (EAT-26) em adolescentes do sexo feminino na cidade de Ribeirão Preto - SP. 2003. 123 páginas. Dissertação (Mestrado em Enfermagem ) - Escola de Enfermagem. USP, Ribeirão Preto, S.P.

BLEICHMAR, E. D. Anorexia/bulimia: um intento de ordenamiento desde el Enfoque Modular-Transformacional. Aperturas psicoanalíticas. Revista I nternacional de Psicoanálisis, Madrid, v. 4, 2000. Disponível em: <http://www.aperturas.org/4diol.html $\geq$. Acesso em: 20 ago. 2007.

BUCARETCHI, H. A. (Org.). Anorexia e Bulimia Nervosa. Uma visão multidisciplinar. São Paulo: Casa do Psicólogo, 2003.

CIRINO, O. Psicanálise e Psiquiatria com crianças. Desenvolvimento ou estrutura. Belo Horizonte: Autêntica, 2001.

CLAUDINO, A. de M.; BORGES, M. B. F. Critérios diagnósticos para os transtornos alimentares: conceitos em evolução. Revista Brasileira de Psiquiatria, São Paulo, v. 24, 2002. Disponível em: <http://www.scielo.br/scielo. php?script=sci_arttext\&pid=S151644462002000700003\&lng=pt\&nrm=iso>. Acesso em: 09 nov. 2006. COBELO, A. W.; GONZAGA, A. P.; NICOLETTI, M. Transtornos Alimentares e seus Aspectos Psicológicos. In: Revista Nutrição Profissional, São Paulo, v. 11, n. 1, p. 35-41, 2007.

CONTI, M. A.; FRUTUOSO, M. F. P.; GAMBARDELLA, A. M. D. Excesso de peso e insatisfação corporal em adolescentes. Revista de Nutrição, Campinas, v. 18, n. 4, p. 491-497, 2005.

CORDÁS, T. A. Transtornos alimentares: classificação e diagnóstico. Revista Psiquiatria Clínica [on line], São Paulo, v. 31, n. 4, p. 154157, 2004. Disponível em: <http://www.scielo.br/scielo.php?>. Acesso em: 05 fev. 2010.

CORDÁS, T. A.; OLIVEIRA, G. K.; FIGUEIREDO, M., DIDONE, N. Transtornos alimentares: Epidemiologia, Etiologia e Classificação. Revista Nutrição Profissional, São Paulo, v. 11 n. 1, p. 12-20, 2007.

DEBORD, G. Sociedade do espetáculo. Paris, Gallimard, 1992.

DUNKER, K. L. L.; PHILIPPI, S. T. Hábitos e comportamentos alimentares de adolescentes com sintomas de anorexia nervosa. Revista de Nutrição, Campinas, v. 16, n. 1, 2003. Disponível em: <http://www.scielo.br/scielo.php?script=sci_arttex,\&pid=S1415- 
$2732003000100006 \&$ Ing=pt\&nrm=iso>. Acesso em: 09 nov. 2006. doi: 10.1590/S1415-52732003000100006.

FERNANDES, M. H. Transtornos alimentares: anorexia e bulimia. São Paulo: Casa do Psicólogo, 2006.

FIATES, G. M. R; SALLES, R. K. Fatores de risco para o desenvolvimento de distúrbios alimentares: um estudo em universitárias. Revista de Nutrição, Campinas, v. 14, supl., p. 3-6, 2001.

FREITAS, S; GOVENSTEIN, C; APPOLINASE, JC. Instrumentos para avaliação dos transtornos alimentares. Revista Brasileira de Psiquiatria, São Paulo, v. 24, supl. 3, p. 34-8, 2002.

FREUD, S. Três ensaios sobre a teoria da sexualidade. (1905). In: Um caso de histeria, três ensaios sobre a sexualidade e outros trabalhos. Rio de Janeiro: Imago, 1974. p. 118-230. (Edição Standard brasileira das obras psicológicas completas de Sigmund Freud, v. 7).

FREUD, S. Sobre o narcisismo: uma introdução. (1914). In:

A história do movimento psicanalítico. Artigos sobre metapsicologia e outros trabalhos. Rio de Janeiro: Imago, 1974/1914, p. 85-119. (Edição Standard Brasileira das Obras Psicológicas Completas de Sigmund Freud, vol. 14).

GARCIA, C. A.; CASTRO, T. G., SOARES, R. M. Comportamento alimentar e imagem corporal entre estudantes de nutrição de uma universidade pública de Porto Alegre - RS. Revista HCPA, Porto Alegre, v. 30, n. 3, 2010.

GARNER, D. M; OLMSTED M P; POLIVY, J. Development and validation of a multidimensional eating disorder inventory for anorexia nervosa and bulimia. I nternational Journal of Eating Disorders I nt. J . Eat Disord, Farmington, v. 2 p. 15-34, 1983.

GREEN, S.; WALTER, P.; KUMAR, V.; KRUST, A.; BONERT, J. M.; ARGOS P. Human oestrogen receptor CDNA: sequence, expression and homology to v-erb-A. Nature, Boston, v. 320, p. 134-139, 1986. KIRSTEN, V. R.; FRATTON, F.; PORTA, N. B. D. Transtornos alimentares em alunas de nutrição do Rio Grande do Sul. Revista de Nutrição, Campinas, v. 22, n. 2, Mar/Abr. 2009.

LACAN, J. O estádio do espelho como formador da função do eu. In: Escritos. Rio de Janeiro: Jorge Zahar, 1998/1949, p. 96-103.

LACAN, J. O Seminário, Livro 4. A relação de objeto, trad. Dulce Duque Estrada, texto estab. por Jacques-Alain Miller. Rio de Janeiro: Zahar, 1995/1956-57, p.254-273.

LASĖGUE, C. De L'anorexie Hystérique. Paris: Études Médicales, 1884. 34p.

MAGALHÂES, V. C.; RAMALHO, R. M. A escuta da palavra silenciada na anorexia e na bulimia. In; SALES, L. (Org.). Pra que essa boca tão grande? Questões acerca da oralidade. Salvador, BA; Ágalma, 2005. 
SANTOS, E.; RIBEIRO, R. P. P.; SANTOS, J. E. D. S; ROSA E SILVA, A. C. J. S; SÁ, M. F. S. Massa óssea em pacientes com anorexia nervosa. Revista Brasileira de Ginecologia e Obstetrícia, Rio de Janeiro, v. 26, n. 1, 2004. Disponível em: $<$ http://www.scielo.br/scielo.php?script=sci_arttext\&pid=S0100$72032004000100011 \& \mathrm{lng}=$ pt\&nrm=iso >. Acesso em: 10 nov. 2006. doi: 10.1590/S0100-72032004000100011.

STIPP, L. M.; OLIVEIRA, M. R. M. Imagem Corporal e Atitudes Alimentares: diferenças entre estudantes de nutrição e de Psicologia. Saúde Revista, Piracicaba, v. 5, n. 9, p. 47-51, 2003.

VILELA, J. E. M; LAMOUNIER, J. A.; FILHO, M. A. D.; BARROS NETO, J. R.; HORTA, G. M. Transtornos alimentares em escolares. J ornal de Pediatria, Rio de Janeiro, v. 80, n. 1, p. 49-54, 2004.

WEI NBERG, C; CORDÁS, T. A; MUNOZ, P. A. Santa Rosa de Lima: uma santa anoréxica na América Latina? Revista de Psiquiatria Clínica do Rio Grande do Sul, Porto Alegre, v. 27, n. 1, 2005. Disponível em: $<$ http://www.scielo.br/scielo.php?script=sci_arttext\&pid=S01018108 $2005000100006 \& \operatorname{lng}=$ pt\&nrm=iso >. Acesso em: 09 nov. 2006.

\section{Endereço para correspondência Nádia Laguárdia de Lima}

Av. Antônio Carlos, 6.627 - Campus Pampulha, 31270-901. Belo Horizonte. Minas Gerais, Brasil.

Endereço eletrônico: nadia.laguardia@gmail.com

\section{Carla de Oliveira Barbosa Rosa}

Universidade Federal de Viçosa - Departamento de Nutrição e Saúde - UFV, 36570000. Viçosa, Minas Gerais

Endereço eletrônico: carla.rosa@ufv.br

José Francisco Vilela Rosa

Av. Silva Lobo, 1730 - Nova Granada. 30340-340. Belo Horizonte, Minas Gerais, Brasil

Endereço eletrônico: zevilela@terra.com.br

Recebido em: 27/01/2011

Reformulado em: 18/05/2011

Aceito para publicação em: 20/05/2011

Acompanhamento do processo editorial: Adriana Benevides Soares

\section{Notas}

* Possui graduação em Psicologia pela Universidade Federal de Minas Gerais, mestrado em Educação pela Universidade Federal de Minas Gerais, doutorado em Educação pela Universidade Federal de Minas Gerais. Atualmente é professora Adjunta do Departamento de Psicologia da Universidade Federal de Minas Gerais.

**Possui graduação em Nutrição pela Universidade Federal de Viçosa (1993), mestrado em Ciência dos Alimentos pela Universidade Federal de Viçosa (1996) e doutorado em Bioquímica Agrícola (2009) pela Universidade Federal de Viçosa. 
Nádia Laguárdia de Lima, Carla de Oliveira Barbosa Rosa, J osé Francisco Vilela Rosa Identificação de fatores de predisposição aos transtornos alimentares

Atualmente é professora adjunta do Departamento de Nutrição e Saúde da Universidade Federal de Viçosa.

***Possui graduação em Engenharia Agrícola pela Universidade Federal de Viçosa (1988), mestrado em Engenharia Agrícola pela Universidade Federal de Viçosa (1995) e doutorado em Engenharia Agrícola (2009) pela Universidade Federal de Viçosa. Tem experiência na área de Probabilidade e Estatística, com ênfase em Probabilidade e Estatística Aplicadas, cálculo e física. Atualmente é professor titular do Centro Universitário Newton Paiva, Belo Horizonte, Brasil. 